\title{
New Seismic Members in NGC 6791 and NGC 6819
}

\author{
B. Bellamy ${ }^{12, a}$ and D. Stello ${ }^{12}$ \\ 1 Sydney Institute for Astronomy (SIfA), University of Sydney, NSW 2006, Australia \\ 2 Stellar Astrophysics Centre (SAC), Aarhus University, 8000 Aarhus C, Denmark
}

\begin{abstract}
We present preliminary results of an asteroseismic analysis of the open clusters NGC 6791 and NGC 6819 in the Kepler field of view. We analysed over 400 stars to extract the asteroseismic properties $v_{\max }$ and $\Delta v$ to make them available to the scientific community. We developed an interactive stitching algorithm that is used to correct for the instrumental drifts in the Kepler light curves and stitch the jumps between each quarter together. We use the SYD pipeline to extract the asteroseismic values and compare these to the expected values for cluster membership to find new member stars for each cluster. We compare our results to previous membership studies.
\end{abstract}

\section{Introduction}

Galactic open star clusters play an important role in constraining our models of stellar evolution. Most stars form in open clusters and disperse into a diversity of field stars in the interstellar medium. Cluster stars therefore provide vital information for acheiving a comprehensive theory of stellar evolution. It is therefore crucial to determine which stars are members of the cluster and which stars are field stars. Stars in clusters are believed to originate from the same interstellar cloud of gas and dust, and hence have some properties in common (age, composition, distance). Cluster membership can therefore be determined using the photometric method [1,2], the kinematic method [3,4], or the asteroseismic method [10] that takes advantage of the properties of the stellar interior. The asteroseismic method has the advantage of being independant of distance and interstellar exinction. The large frequency separation, $\Delta v$, between modes of the same degree and consecutive orders depend on the mean density of the star, and the frequency of maximum power, $v_{\max }$, is related to the surface gravity and effective temperature. Both of these properties are known to scale with $\mathrm{M}, \mathrm{L}$ and $\mathrm{T}_{\text {eff }}$ of the star [5,6], thus we can use these relationships to infer these properties without the need for detailed modelling for each star $[7,8]$. The completion of the Kepler mission has provided 4 times longer time series data than used in similar stiudies in previous work [9], with 4 years of almost continuous photometric data. We are therefore able to measure the asteroseismic properties, $v_{\max }$ and $\Delta v$, in most of the red giants in the field of view of NGC 6791 and NGC 6819.

\section{Determination of membership status}

We used the Kepler data obtained through various Kepler Asteroseismic Science Consortium (KASC) and Guest Observer (GO) proposals, providing a total 407 stars within a 20 arcmin radius of the clusters. All available data was collected and analysed using our interactive stiching program. The interactive program used one of three simple sticthing algorithms to correct for the characterstic kepler jumps between each quarter. Instrumental trends were corrected for using smoothing and polynomial fits of varying orders.

a e-mail: bellamy@physics.usyd.edu.au

This is an Open Access article distributed under the terms of the Creative Commons Attribution License 4.0, which permits unrestricted use, distribution, and reproduction in any medium, provided the original work is properly cited. 

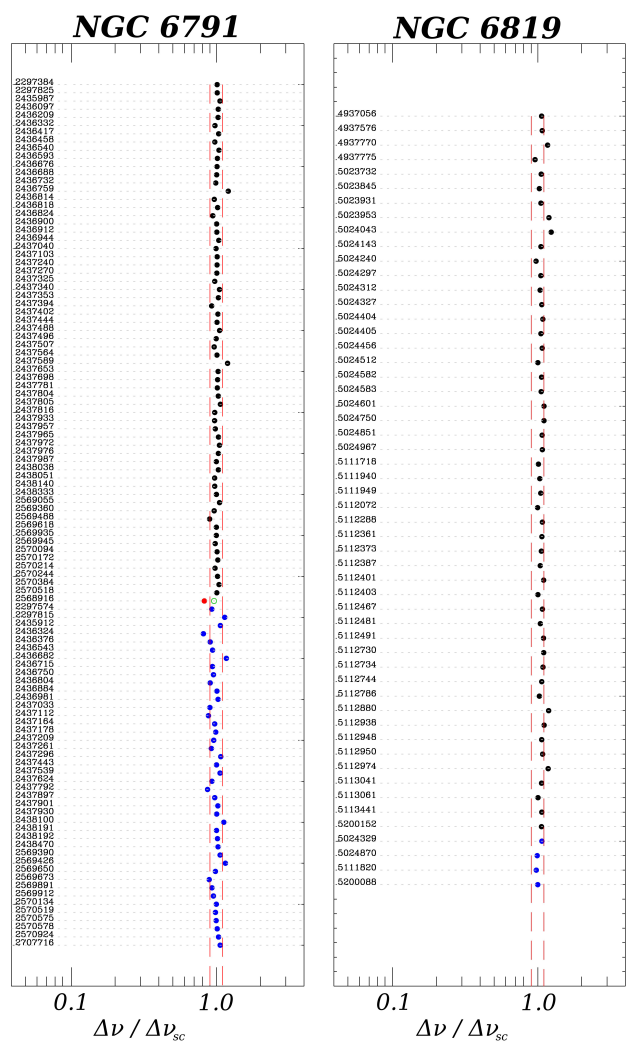

Fig. 1. Cluster members of the two clusters identified by the ratio of the measured asteroseismic parameters to the solar scaled parameters. Only the $\Delta v$ ratios are shown for simplicity. The vertical dashed lines are indicative of $2 \sigma$ from the expected ratio of 1 . The black dots indicate members from [9] that are confirmed in this work. The red dots indicate members from [9] that we could not confirm with certainty (We note that [9] use only one quarter, Q4, for KIC 2568916). The blue dots indicate likely new members found by this work.

Using the approach of [10], we use asteroseismic measurements of $v_{\max }$, and $\Delta v$ to determine cluster membership of our selected stars. The SYD pipeline was used to extract the asteroseismic properties $v_{\text {max }}$, and $\Delta v$. A detailed description of the SYD pipeline can be found in [11]. Comparison of these values with the expected solar scaled values allows us to infer the cluster membership. The observed-to-expected ratios for $\Delta v$ and $v_{\max }$ are shown in Figure 1. The expected ratio is 1.0 with a $10 \%$ uncertainty, marked by the red lines.

\section{References}

1. Stetson, P. B et. al. PASP, 115, (2003), 413-447

2. Brogaard, K. et. al. A\&A, 543, (2012), A106

3. Hole, K. T. et. al. AJ, 138, (2012), 159-168

4. Gao, X.-H, Research in Astronomy and Astrophysics, 14, (2014), 159-164

5. Ulrich, R. K. ApJ, 306, (1986), L37-L40

6. Kjeldsen, H. and Bedding, T. R. A\&A, 293, (1995), 86-106

7. Stello, D. and Bruntt, H. and Preston, H. and Buzasi, D. ApJ, 674, (2008), L53-L56

8. Kallinger, T. et. al. A\&A, 522, (2010), A1

9. Stello, D. et. al. ApJ, 739, (2011), 13

10. Stello, D. et. al. ApJ, 713, (2010), L182-L186

11. Huber, D et. al. Comm. Astron., 160, (2009), 74 